\title{
Groundwater response to tidal forcing
}

\author{
Melanie E. Roberts ${ }^{1}$
}

(Received 7 August 2008; revised 22 December 2008)

\begin{abstract}
The groundwater response to tidal forcing in a phreatic aquifer is modelled using a perturbation approach for the case of a shallow beach. The principal features of the groundwater response, namely the super-elevation of the water table, attenuation of the forcing wave and the asymmetry and phase shift in the fluctuations, is investigated and compared with earlier solutions. These results demonstrate the importance of correctly including the beach slope effects to prevent the exaggeration of observed features for shallow beaches. A general solution method is presented, which provides an efficient method for obtaining higher order solutions.
\end{abstract}

\section{Contents}

\section{Introduction}

http://anziamj . austms.org. au/ojs/index.php/ANZIAMJ/article/view/1401 gives this article, (c) Austral. Mathematical Soc. 2008. Published December 24, 2008. ISSN 1446-8735. (Print two pages per sheet of paper.) 
2 The model

C642

3 Solution method

C646

4 Results

C648

5 Discussion

C650

References

C653

\section{Introduction}

The tidal forcing of phreatic aquifers induces a wave that propagates through the aquifer. As the near shore region of a coastal aquifer is an area of high biological and mechanical activity, a thorough understanding of the interaction of tides and the groundwater table is desirable. Specifically, tidal forcing has been shown to influence beach erosion and aggregation, saltwater intrusion and chemical transport in addition to the groundwater table fluctuations [1].

Philip [3] modelled the groundwater response to tidal forcing for a vertical beach and found that the water table is elevated above mean sea level even in the absence of recharge. Nielsen [2] further investigated the inland overheight by considering the effects of a sloping beach via a perturbation solution. His result, given to second order, demonstrated that a sloping beach enhanced the inland overheight as well as the asymmetry in the water table fluctuations and their shift from the tidal signature.

Nielsen employed the Boussinesq equation to model the groundwater response, which arises from the Dupuit approximation of dominating horizontal flow. Numerical modelling by Ataie-Ashtiani et al. [1] indicates that under certain conditions vertical flows are significant. The effect of vertical flows on the groundwater response was investigated by Teo et al. [4] using pertur- 
bation expansions under the assumptions of a shallow aquifer and small tidal amplitude. Although Teo et al. and Nielsen gave their solutions for beach slopes in the range $0-\pi / 2$, the nature of the perturbation expansions is such that for sufficiently shallow beaches the solutions exaggerate the observed features and indicate unphysical behaviour. Thus, we are motivated to consider the groundwater response to tidal forcing across a shallow beach. Teo et al.'s solution method is extended with an appropriate re-arrangement of the order equations to account for a shallow beach. A general solution method, which was automated in Mathematica, is presented and this approach enables the solution to be continued to higher order than previously reported.

\section{The model}

A homogeneous, isotropic and rigid porous medium with a phreatic surface is connected to a tidal surface water body through a sloping beach. The beachface, which is of constant slope, shares the same porous medium properties as the aquifer. For simplicity, we assume that the surface and groundwater are of the same constant density and that the water table is connected to the tide at the beach-face. As shown in Figure 1, the base of the aquifer is horizontal and impervious, extends semi-infinitely inland, and is invariant along the beach-front. In addition, we assume that a sharp interface exists between the water table and the unsaturated porous medium above, that is, we neglect capillarity.

Coupling of the Darcy flux equation with the continuity equation for a saturated porous medium with constant density gives rise to Laplace's equation in the potential head, $\phi=z+p / \rho g$, namely,

$$
\nabla^{2} \phi=0,
$$

where $p$ is the pore-water pressure, $\rho$ is the density, and $g$ is gravitational acceleration. Equation (1) is to be solved subject to appropriate boundary 


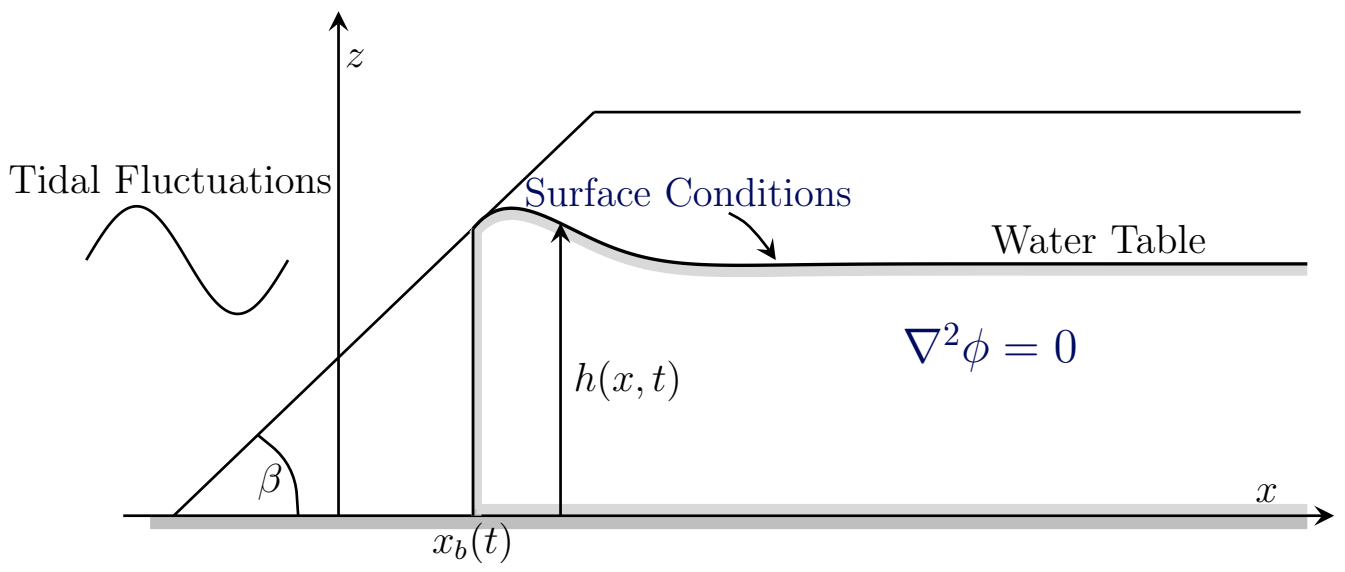

Figure 1: Geometry of the aquifer. Laplace's equation is solved in the region bounded by the horizontal extent of the tide $x_{b}(t)$, the base of the aquifer $z=0$, and the water table $z=h(x, t)$. The horizontal axis is chosen such that $x=0$ corresponds to the low tide line.

conditions. The surface conditions, which are applied at the unknown surface of the water table, $h(x, t)$, arise from the requirement that the pore-water pressure is atmospheric on the water table, and the assumption that particles once on the surface remain there. Then

$$
\left.\begin{array}{l}
\phi=h \\
n_{e} \phi_{t}=K\left(\phi_{x}^{2}+\phi_{z}^{2}\right)-K \phi_{z}
\end{array}\right\} \quad z=h(x, t),
$$

where $n_{e}$ is the effective porosity and $K$ is the hydraulic conductivity. Given the above constraints, the impervious aquifer base means that

$$
\phi_{z}=0 \quad \text { on } \quad z=0 \text {, }
$$

and the height of the water table far inland is an as yet unknown constant SO

$$
\phi_{x} \rightarrow 0 \text { as } \quad x \rightarrow \infty
$$


For a sinusoidally varying tide at a beach of constant slope $\beta$ the horizontal extent of the shoreline is $x_{b}(t)=(D+A \cos \omega t) \cot \beta$, recalling that $x=0$ corresponds to the low tide line (refer to Figure 1). Under the assumption that the water table is coupled to the tide at the shoreline, which prevents the formation of a seepage face, then the seaward boundary condition is

$$
h\left(x_{b}(t), t\right)=D+A \cos \omega t
$$

where $\mathrm{D}$ is the undisturbed depth of the aquifer, $\mathrm{A}$ is the tidal amplitude, and $\omega$ is the tidal frequency.

In the vertical direction we choose to scale by the undisturbed aquifer depth $\mathrm{D}$, and in the horizontal direction by the characteristic length of decay [2]

$$
L=\sqrt{\frac{2 K D}{n_{e} \omega}},
$$

with time scaled by the inverse tidal frequency $1 / \omega$. We therefore set

$$
x^{\prime}=\frac{x}{\mathrm{~L}}, \quad z^{\prime}=\frac{z}{\mathrm{D}}, \quad \mathrm{h}^{\prime}=\frac{\mathrm{h}}{\mathrm{D}}, \quad \phi^{\prime}=\frac{\phi}{\mathrm{D}} \quad \text { and } \quad \mathrm{t}^{\prime}=\mathrm{t} \omega,
$$

to obtain the system of equations in dimensionless form.

The seaward and surface boundary conditions are enforced at time dependent locations, moreover, the position of the water table, $h(x, t)$, must be obtained as part of the solution. We therefore transform the equations onto a fixed domain by setting

$$
x^{\prime \prime}=x^{\prime}-x_{b}^{\prime}\left(t^{\prime}\right)=x^{\prime}-\alpha \varepsilon \cot \beta
$$

and

$$
z^{\prime \prime}=\frac{z^{\prime}}{h^{\prime}\left(x^{\prime}, t^{\prime}\right)}
$$

where $\alpha=A / D$ is the small tidal amplitude parameter and $\varepsilon=D / L$ is the shallow aquifer parameter. 
On dropping the dash notation the system of equations is

$$
\begin{aligned}
& \phi_{z z}+\varepsilon^{2}\left(h^{2} \phi_{x x}-2 z h h_{x} \phi_{x z}+z^{2} h_{x}^{2} \phi_{z z}\right)=0, \\
& h(0, t)=1+\alpha \cos (\omega t), \\
& \phi_{x}=0 \text { as } x \rightarrow \infty, \\
& \phi_{z}=0 \text { on } z=0, \\
& \phi=h \quad \text { on } z=1, \\
& 2 \varepsilon^{2}\left[\phi_{t} h^{2}+h^{2} \phi_{x} \alpha \varepsilon \cot \beta \sin t-h \phi_{z}\left(h_{t}+h_{x} \alpha \varepsilon \cot \beta \sin t\right)\right] \\
& \quad-\varepsilon^{2}\left(h \phi_{x}-z h_{x} \phi_{z}\right)^{2}-\phi_{z}^{2}+h \phi_{z}=0 \quad \text { on } z=1,
\end{aligned}
$$

which is to be solved on the domain $0 \leq x<\infty, 0 \leq z \leq 1$ with $\mathrm{t} \geq 0$.

The application of the boundary fixing transformations (8) introduces the beach slope, $\beta$, into the surface boundary condition explicitly through the term $\alpha \varepsilon \cot \beta \sin t$, as seen in (14). For $\beta \sim \mathrm{O}(1)$, this term is $\mathrm{O}(\alpha \varepsilon)$; however, for sufficiently shallow beaches $\cot \beta$ becomes large and it is necessary to consider the magnitude of this term.

For $\beta \sim O(\varepsilon)$, we make the approximation

$$
\beta=\beta_{0} \varepsilon,
$$

thus $\cot \beta \approx\left(\beta_{\circ} \varepsilon\right)^{-1}-\frac{1}{3} \beta_{0} \varepsilon$, and the surface boundary equation (14) becomes

$$
\begin{aligned}
2 \varepsilon^{2}\left[\phi_{\mathrm{t}} h^{2}+h^{2} \phi_{x}\right. & \alpha \sin t\left(\frac{1}{\beta_{0}}-\frac{\beta_{0} \varepsilon^{2}}{3}\right) \\
& \left.-h \phi_{z}\left(h_{\mathrm{t}}+h_{x} \alpha \sin t\left(\frac{1}{\beta_{0}}-\frac{\beta_{0} \varepsilon^{2}}{3}\right)\right)\right] \\
& -\varepsilon^{2}\left(h \phi_{x}-z h_{x} \phi_{z}\right)^{2}-\phi_{z}^{2}+h \phi_{z}=0 \text { on } z=1 .
\end{aligned}
$$




\section{Solution method}

The nonlinear nature of the system of equations (9)-(13) with (16) is such that an exact solution is not possible. Teo et al. [4] considered hydraulic conductivities in the range 50-1000 m/day, with aquifer depths of 5-10 m and tidal amplitudes of 1-2 m, while Nielsen [2] considered tidal amplitudes and aquifer depths on the order of $0.5 \mathrm{~m}$, with hydraulic conductivities on the order of a few $\mathrm{mm} /$ day. Given a soil porosity in the range $0.2-0.4$ and considering semi-diurnal and diurnal tides, these values correspond to parameter values in the range 0.1 to 0.6 . We therefore seek solutions for the case $\varepsilon \ll 1$ and $\alpha \ll 1$.

A series solution for $\phi(x, z, t)$ in terms of $h(x, t)$ is obtained by setting

$$
\phi(x, z, t)=\sum_{i=0}^{\infty} \varepsilon^{i} \phi_{i}(x, z, t) \text { and } h(x, t)=\sum_{i=0}^{\infty} \varepsilon^{i} h_{i}(x, t) .
$$

Solution of (9) with (17) subject to the aquifer base condition (12) and the surface condition (13) at the first three orders gives

$$
\phi_{0}(x, z, t)=h_{0}(x, t), \quad \phi_{1}(x, z, t)=h_{1}(x, t)
$$

and

$$
\phi_{2}(x, z, t)=\left(1-z^{2}\right)\left(h_{0} h_{1} h_{0 x x}+\frac{1}{2} h_{0}^{2} h_{1 x}\right)-h_{2}(x, t) .
$$

Application of the expressions in $\phi_{i}(x, z, t)$ to the surface condition (14) gives rise to a series of partial differential equations in $h_{\mathfrak{i}}(x, t)$. The leading order equation is

$$
2 h_{0 t}-h_{0 x}^{2}-h_{0} h_{0 x x}+\frac{2 \alpha \sin t}{\beta_{0}} h_{0 x}=0,
$$

which is of nonlinear parabolic type. At higher orders the general form is

$$
h_{i t}-\frac{1}{2} h_{0} h_{i x x}+\frac{2 h_{i} h_{0 t}}{h 0}-h_{0 x} h_{i x}-\frac{3}{2} h_{i} h_{0 x x}
$$




$$
-\frac{h_{i} h_{0 x}^{2}}{h_{0}}+\frac{h_{i x} \alpha \sin t}{\beta_{0}}+\frac{2 h_{i} h_{0 x} \alpha \sin t}{\beta_{0} h_{0}}=\text { RHS }
$$

where the forcing term RHS is a function of the lower order solutions $h_{i-1}(x, t)$, $h_{i-2}(x, t), \ldots, h_{0}(x, t)$. The system of equations (21), with the exception of (20), are variable coefficient, forced, linear equations that cannot be solved exactly.

As we are predominantly interested in small $\alpha$, we seek series solutions to (20) and (21) setting

$$
h_{i}(x, t)=\sum_{j=0}^{\infty} \alpha^{j} h_{i, j}(x, t) .
$$

Application of (22) to (20) and (21) results in a series of partial differential equations of the form

$$
2 h_{i t}-h_{i x x}=f(x, t),
$$

where $f(x, t)$ is a known function of the earlier terms. The series of partial differential equations (23) is solved order by order subject to the tidal boundary condition (10) and inland condition (11).

While the solution to the first few orders of (23) can be obtained quickly using any in-built solver package, such as Mathematica's DSolve command, at higher orders this becomes cumbersome and takes increasingly long times. We therefore use the known structure of (23) to improve the efficiency of this procedure. Since $f(x, t)$ in $(23)$ is of the form

$$
\sum_{n}\left(a_{0}+a_{1} x+\cdots+a_{k} x^{k}\right) e^{b x+c} e^{i n(t-x)}
$$

with constants $a_{k}, b$ and $c$, we determine a general solution to (23) to within a number of constants, and then solve for the unknown constants by substituting (24) into (23). This method improved the efficiency and memory requirements by $80 \%$. 


\section{Results}

To fourth order, the water table height is

$$
h(x, t)=\sum_{i}^{4} \sum_{j}^{4} \alpha^{j} \varepsilon^{i} h_{i, j}(x, t), \quad i+j \leq 4,
$$

and the error associated with this solution is the greatest of $O\left(\varepsilon^{i} \alpha^{j}\right), i+j=5$. To second order the solution is

$$
\begin{aligned}
h(x, t)=1+\alpha & e^{-x} \cos (t-x)+\frac{\alpha^{2}}{4}\left\{1+\frac{2}{\beta_{0}}-e^{-2 x}\right. \\
- & 2 e^{-2 x} \cos 2(t-x)+2 e^{-\sqrt{2} x} \cos (2 t-\sqrt{2} x) \\
& +\frac{e^{\sqrt{2} x}}{\beta_{0}}[\cos (2 t+\sqrt{2} x)+\cos (2 t-\sqrt{2} x) \\
& +2 \sin (2 t+\sqrt{2} x)-2 \sin (2 t-\sqrt{2} x)] \\
- & \left.\frac{2 e^{-x}}{\beta_{0}}[\cos x+\sin x+\cos (2 t-x)-\sin (2 t-x)]\right\} .
\end{aligned}
$$

The principal features contained in this solution are an exponentially decaying wave, which shifts from the tidal signature as it propagates through the aquifer, the elevation of the water table above mean sea level and the asymmetry in the local fluctuations of the water table. We note that a minor error occurs in Teo et al.'s solution at their Equation (17). As this error propagates to their conclusion, we refer here to the corrected Teo et al. solution without further comment.

The constant term in the solution (26), $\alpha^{2} / 4$, indicates that the water table is elevated above mean sea level. This elevation of the water table is known as the inland overheight, which was first observed by Philip [3]. To 
fourth order, the inland overheight is

$$
\frac{1}{4} \alpha^{2}-\frac{1}{32} \alpha^{4}+\frac{1}{2} \alpha^{2} \beta_{0}^{-1}-\left(\frac{6+5 \sqrt{2}}{80 \beta_{0}}+\frac{2+\sqrt{2}}{16 \beta_{0}^{2}}+\frac{-1+\sqrt{2}}{4 \beta_{0}^{3}}\right),
$$

which agrees with Philip's solution to first order. The inland overheight is a result of the greater effective transmissivity of the aquifer at high tide. During the rising tide, water flows landward at a faster rate than it flows seaward during the ebb tide, which is balanced by an elevation in the water table; thus a greater tidal amplitude and hydraulic conductivity, which arises implicitly through $\beta_{0}$, see (15), enhances the extent of the overheight, while a deeper aquifer mitigates this effect. A sloping beach enhances the overheight as water can infiltrate into the aquifer vertically during the rising tide, but is only able to seep from the aquifer horizontally during the ebb [2].

Discussion of the overheight is generally confined to the inland overheight, the elevation of the water table far from the beach; however, the mean water table height is neither the undisturbed aquifer depth or the inland overheight, but rather increases from mean sea level through the intermediate region of the aquifer before relaxing to the inland overheight. Comparison of the mean water table heights given by (26), and Teo et al. and Nielsen's solutions indicates that failure to include the beach slope effects at the correct order results in the mean water table height being over-predicted. Nielsen's solution elicits a constant mean water table height due to the use of a matching condition at the seaward boundary, which applied the tidal condition at a fixed location.

The difference between the inland overheight and the mean water table height indicates an asymmetry in the local fluctuations of the water table, which arises as the porous medium fills faster than it drains. The asymmetry in the water table fluctuations increases with distance into the aquifer, as shown in Figure 2, and with a greater tidal amplitude or hydraulic conductivity. As with the inland overheight, a deeper aquifer mitigates these effects. The sloping beach also gives rise to a greater asymmetry in the water table 
fluctuations due to the migration of the forcing point inland during the rising tide in addition to the horizontal infiltration of water over this period. The slight elevation in the water table corresponding to low tide in Teo et al.'s solution, and the fast drainage of the aquifer in Nielsen's solution are not expected, and indicate that these solutions have probably been applied outside of their range of validity.

The asymmetry in the fluctuations is investigated via harmonic analysis. Decomposing the solution (26) as $h(x, t)=\sum_{m} C_{m}(x) e^{i m x}$, where $C_{m}(x)$ is the coefficient associated with the mth complex Fourier function, the magnitude of the mth harmonic is $2 \sqrt{C_{m} C_{-m}}$.

The primary harmonic was not significantly influenced by the beach slope, whereas the secondary harmonic increases in magnitude with a shallower beach-particularly through the intermediate region of the beach. While the primary harmonic is dominant, and is the slowest to decay, the ratio of the primary and secondary harmonics decreases with distance into the aquifer indicating that the influence of higher order harmonics also increases with distance into the aquifer. This is seen through the increase in the asymmetry of the water table fluctuations at locations further from the beach, see Figure 2.

\section{Discussion}

The groundwater response to tidal forcing across a sloping beach has been described in terms of a shallow aquifer and a small tidal amplitude parameter together with the inclination of the beach to the horizontal. It has been shown how the re-ordering of the governing equations with respect to the beach slope provides for an improved description of the water table response for sufficiently shallow beaches, as use of the non-corrected equations can give misleading information about the water table dynamics. This fourth order perturbation solution confirms that shallower beaches enhance the features of 


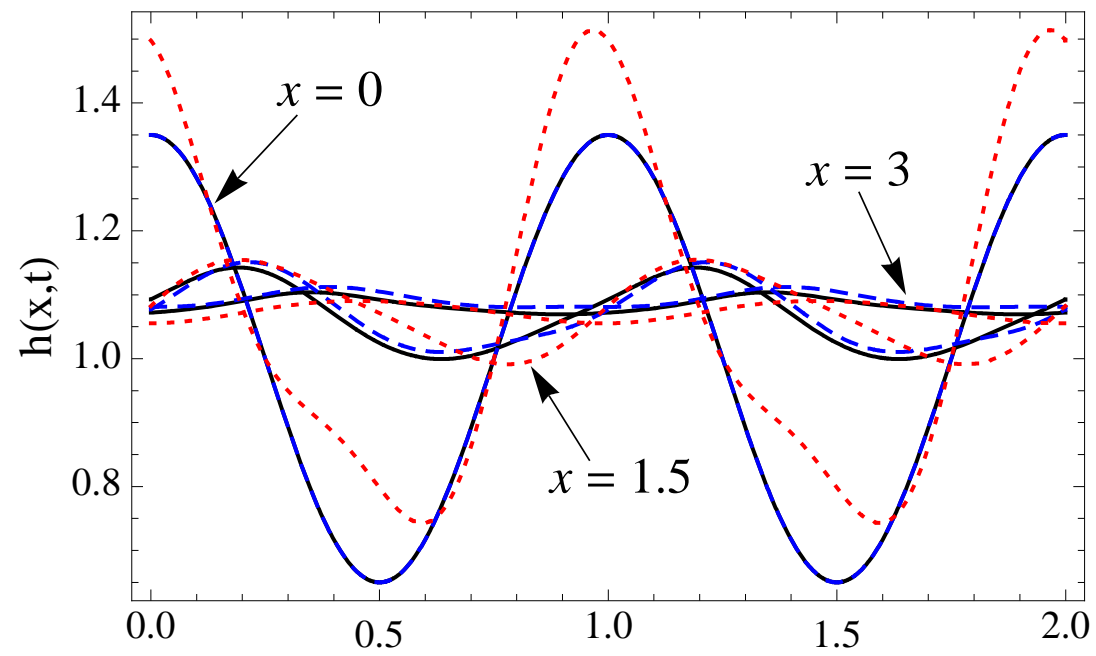

(a)

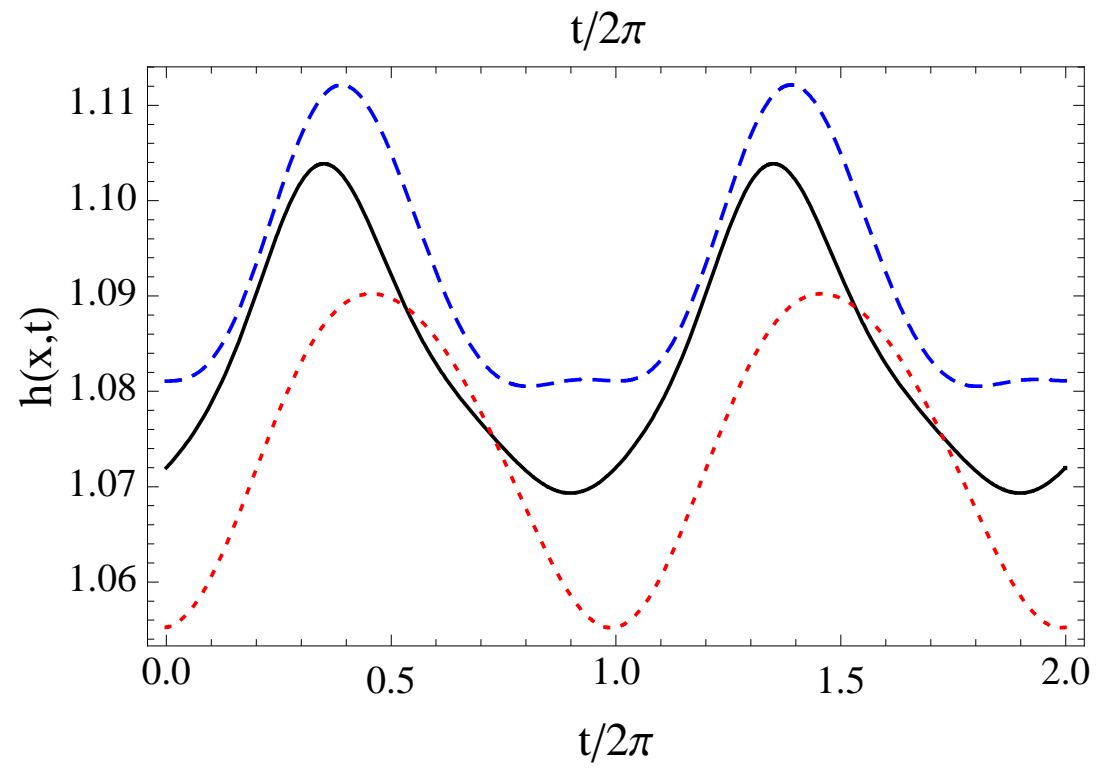

(b)

FiguRE 2: Local fluctuations of the water table over two tidal periods as given by (26) (black/solid), Teo et al.'s solution (blue/dashed) and Nielsen's solution (red/dotted). The variation is given at (a) the beach-face, $x=0$, and at $x=1.5$ and $x=3$ and (b) $x=3$, with a beach-slope of $\beta=\pi / 8$ and $\alpha=0.35$ and $\varepsilon=0.5$. 
the aquifer: the inland overheight and super-elevation of the water table, the asymmetry of the local fluctuations, and the shift of the fluctuations away from the tidal signature.

One important component of this work has been the analysis of the role played by higher order harmonics. The relative magnitude of higher harmonics to lower order harmonics increases with distance into the aquifer for shallow beaches, which is evident in the increased asymmetry in the local water table fluctuations further from the shoreline, and results from the imbalance in the pore drainage. In addition to causing the asymmetry in the water table fluctuations, the imbalance in the pore drainage results in an elevation of the mean water table height, as well as the height of the water table far inland.

Comparison of these features with previous solutions demonstrates that failure to account for the growth of $\cot \beta$ with shallow beaches in the perturbation solution exaggerates the water table response and give misleading information. The use of the known structure of the solution to automate the solution procedure has enabled the solution to be presented to higher orders than previously.

The current work is specific to shallow beaches. It is of interest to consider the groundwater table response for the full range of beach slopes. The above method has been employed to examine the results of tidal forcing at beaches of both steep and shallow slope, the results of which will be reported elsewhere.

Acknowledgements I am grateful to Dr. Paul Abbott (Physics UWA) for his assistance with the computer algebra, and Prof. Andrew Bassom (Maths UWA), Dr. Neville Fowkes (Maths UWA) and Dr. Mike Trefry (CSIRO Floreat) for their assistance with the solution method. I thank CSIRO and ANZIAM for the provision of funding to attend the CTAC08 conference. 


\section{References}

[1] B. Ataie-Ashtiani, R. E. Volker, and D. A. Lockington. Tidal effects on groundwater dynamics in unconfined aquifers. Hydrological Processes, 15:655-669, 2001. doi:10.1002/hyp.183 C641

[2] P. Nielsen. Tidal dynamics of the water table in beaches. Water Resour. Res., 26(9):2127-2134, September 1990. C641, C644, C646, C649

[3] J.R. Philip. Periodic nonlinear diffusion: An integral relation and its physical consequences. Aust. J. Phys., 26:513-519, 1973. C641, C648

[4] H. T. Teo, D. S. Jeng, B. R. Seymour, D. A. Barry, and L. Li. A new analytical solution for water table fluctuations in coastal aquifers with sloping beaches. Adv. Wat. Res., 26:1239-1247, December 2003. doi:10.1016/j.advwatres.2003.08.004 C641, C646

\section{Author address}

1. Melanie E. Roberts, School Maths \& Statistics, University of Western Australia, Australia.

mailto:melanie@maths.uwa.edu . au 